\title{
ERRATA
}

\section{COMMENTS ON "INTERACTING QUANTUM FIELDS"}

\author{
[Rev. in Maths., Vol. 11, No. 7 (1999) 881-928 \\ Glenn Eric Johnson \\ Litton-TASC, 4801 Stonecroft Blvd. \\ Chantilly, VA 20151, USA
}

The conclusions that Schwinger functions constructed from generalized random processes defined on sampling functions imply quantum fields with scattering is not supported by the arguments within [1]. The constructed Schwinger functions do analytically continue to define Wightman functions $[1,2]$. These Wightman functions exhibit scattering with cross sections and equivalent potentials calculated in [1]. Nevertheless, there are two deficiencies in the arguments of [1] stating that there are quantum fields with the defined scattering amplitudes defined in spaces with positive metric. Development of scattering for quantum fields defined in spaces with indefinite metric is discussed in $[2,3]$ and their further references. The nonvanishing scattering amplitudes correspond to boundary values of the constructed analytic Wightman functions that are approached from beyond the algebra of functions $\mathcal{E}_{+}$within which positivity is demonstrated. $\mathcal{E}_{+}$is the algebra of sampling functions constructed in [1] for which the Schwinger functions define a nonnegative reflection (Osterwalder-Schrader [4]) positive form. Hence, the demonstrated nonnegativity results do not directly apply to the nontrivial scattering amplitudes. Indeed, the nonvanishing truncated four-point function corresponding to

$$
\left\langle a^{*}\left(g_{1}, t_{1}\right) a^{*}\left(g_{2}, t_{2}\right) \Omega, a^{*}\left(g_{3}, t_{3}\right) a^{*}\left(g_{4}, t_{4}\right) \Omega\right\rangle^{T}
$$

is a boundary value at vanishing Euclidean times approached from (just) beyond $\mathcal{E}_{+}$. The boundary values approachable from within $\mathcal{E}_{+}$exhibit no scattering. This nontriviality makes these models interesting, but requires a proof of positivity that extends beyond $\mathcal{E}_{+}$, i.e. more than the "factor" positivity of [1].

Secondly, to get a representation in a Hilbert space of these field operators requires a demonstration that the null space is an ideal. There is a Hilbert space representation of states, but the accepted definition for a field operator within the 
Hilbert space fails unless the null space is an ideal. The limited algebra of sampling functions $\mathcal{E}_{+}$for which the Wightman functions define a nonnegative bilinear functional requires an explicit demonstration that the null space is an ideal since the algebra cannot be self-dual, i.e. a $*$-algebra.

The issue of boundary values can be illustrated with the two-point function of the model constructed on $\mathcal{E}_{+}$.

$$
\begin{aligned}
&{ }^{T} S_{2}\left(\vartheta \varphi_{1}^{*}, \varphi_{2}\right) \\
&=\int d \xi\left(R * \vartheta \varphi_{1}^{*}\right)\left(R * \varphi_{2}\right) \\
&=\int d \tau_{1} d \tau_{2} \int d \mathbf{p} \frac{\pi}{2 \omega_{m}}\left(-\frac{d}{d \omega_{m}}\right)\left(\frac{e^{-\omega_{m}\left|\tau_{1}-\tau_{2}\right|}}{\omega_{p}}\right) \\
& \cdot h_{1}(\mathbf{p})^{*}\left(\omega_{m} \delta\left(-\tau_{1}-\tau_{1}^{\prime}\right)-\delta^{\prime}\left(-\tau_{1}-\tau_{1}^{\prime}\right)\right) h_{2}(\mathbf{p})\left(\omega_{m} \delta\left(\tau_{2}-\tau_{2}^{\prime}\right)-\delta^{\prime}\left(\tau_{2}-\tau_{2}^{\prime}\right)\right) \\
&=\left.\int d \mathbf{p} \frac{\pi}{2 \omega_{m}} h_{1}(\mathbf{p})^{*} h_{2}(\mathbf{p})\left(\omega_{m}+\frac{d}{d \tau_{1}^{\prime}}\right)\left(\omega_{m}+\frac{d}{d \tau_{2}^{\prime}}\right)\left(-\frac{d}{d \lambda}\right) \frac{e^{-\lambda\left|\tau_{1}^{\prime}+\tau_{2}^{\prime}\right|}}{\lambda}\right|_{\lambda=\omega_{m}} \\
&=\left.\int d \mathbf{p} \frac{\pi}{2 \omega_{m}} h_{1}(\mathbf{p})^{*} h_{2}(\mathbf{p})\left(-\frac{d}{d \lambda}\right)\left(\left(\omega_{m}-\lambda \operatorname{sgn}\left(\tau_{1}^{\prime}+\tau_{2}^{\prime}\right)\right)^{2} \frac{e^{-\lambda\left|\tau_{1}^{\prime}+\tau_{2}^{\prime}\right|}}{\lambda}\right)\right|_{\lambda=\omega_{m}} \\
&= \int d \mathbf{p} h_{1}(\mathbf{p})^{*} h_{2}(\mathbf{p})\left\{\begin{array}{l}
0 \\
2 \pi\left|\tau_{1}^{\prime}+\tau_{2}^{\prime}\right| e^{-\omega_{m}\left|\tau_{1}^{\prime}+\tau_{2}^{\prime}\right|} \\
\tau_{1}^{\prime}+\tau_{2}^{\prime}<0 .
\end{array}\right.
\end{aligned}
$$

The boundary value approached from $\tau_{1}^{\prime}+\tau_{2}^{\prime}<0$ is beyond $\mathcal{E}_{+}$since $\tau_{k}^{\prime} \geq 0$ for $\varphi_{k} \in \mathcal{E}_{+}$. Only the analytic extension of this boundary value is nontrivial. The desired field ordering corresponding to $\tau_{1}>\tau_{2}$ also cannot be achieved while remaining within $\mathcal{E}_{+}$. This two-point function is nonnegative for $\varphi_{1}=\varphi_{2}$, and its analytic extension diverges as $\left|t_{1}-t_{2}\right|$ on the nontrivial boundary (i.e. at physical times).

It is currently speculative whether the augmented models of [1]] define nontrivial quantum fields on spaces with positive metric. To encompass the non-trivial scattering amplitudes, the nonnegativity demonstrated for $\mathcal{E}_{+}$needs to be extended to at least $\mathcal{E}_{0^{-}}$, sampling functions with support for Euclidean times $\tau \geq-\epsilon$ for a real $\epsilon>0$.

\section{Acknowledgment}

I would like to thank Dr. H. Gottschalk for many insightful comments and correspondence on the problems with [1].

\section{References}

[1] G. Johnson, "Interacting quantum fields", Rev. Math. Phys. 11 (1999) 881-928.

[2] S. Albeverio, H. Gottschalk and J.-L. Wu, "Convoluted generalized white noise, Schwinger functions and their analytic continuation to Wightman functions", Rev. Math. Phys. 8 (1996) 763-817. 
[3] S. Albeverio, H. Gottschalk and J.-L. Wu, "Nontrivial scattering amplitudes for some local relativistic quantum field models with indefinite metric", Phys. Lett. B405 (1997) 243.

[4] K. Osterwalder and R. Schrader, "Axioms for Euclidean Green's Functions", Commun. Math. Phys. 31 (1973) 83-112. 